\title{
Preliminary Note on the Reduction of Measures of Photographic
} Plates. By H. H. Turner, M.A., B.Sc.

1. The following is an attempt to simplify the processes already suggested for the reduction of measures of stellar photographs, and especially measures of the plates of the Astrographic Chart.

2. The fundamental property of a photographic plate is that it is a projection of the celestial sphere from a point (the centre of the object-glass) on a plane (the photographic plate itself). This statement must be qualified just so far as the image of the star is displaced by-.

(a) Distortion of the objective;

(b) Displacements of the photographic film;

(c) Refraction and aberration.

3. Neglecting for the moment these disturbing causes, it follows that any great circle on the sphere projects into a straight line on the plate; and any straight line on the plate corresponds to a great circle on the sphere, and hence to a straight line on any other plate containing the same region.

4. The appropriateness of using rectilinear co-ordinates is thus suggested : for the transformation from any rectilinear co-ordinates on one plate to any rectilinear on another takes a simple form. Let $(x, y),(\xi, \eta)$ be any rectilinear co-ordinates of the same point on the two plates; then the following general relations hold :

$$
\xi=\frac{\mathrm{A} x+\mathrm{B} y+\mathrm{C}}{\mathrm{K} x+\mathrm{L} y+\mathrm{M}}, \quad \eta=\frac{\mathrm{D} x+\mathrm{E} y+\mathrm{F}}{\mathrm{K} x+\mathrm{L} y+\mathrm{M}} .
$$

It is readily seen that the denominator must be the same in the two cases, for the equation

$$
\lambda \xi+\mu \eta+\nu=0
$$

represents a straight line on one plate for all valnes of $\lambda, \mu, \nu$, and hence must represent a straight line on the other. The above formulæ give the equation to this straight line, viz.

$$
\lambda(\mathrm{A} x+\mathrm{B} y+\mathrm{C})+\mu(\mathrm{D} x+\mathrm{E} y+\mathrm{F})+\nu(\mathrm{K} x+\mathrm{L} y+\mathrm{M})=0 .
$$

5. The above formulæ are quite general, but a more convenient form may be given to them in the case of co-ordinates measured approximately in similar directions on the two plates, as will generally be the case for the plates of the Astrographic Chart. We shall then have approximately

$$
\xi=x+\text { const., } \quad \eta=y+\text { const. },
$$


and the accurate relations may be written

$$
\xi=\frac{(\mathrm{I}+a) x+b y+c}{\mathrm{I}-k x-l y}, \quad \eta=\frac{d x+(\mathrm{I}+e) y+f}{\mathrm{I}-k x-l y},
$$

where $a, b, d, e, k, l$ are all small, and $c$ and $f$ are the co-ordinates of the centre of the plate $(x, y)$ on the plate $(\xi, \eta)$, which are about $\mathbf{I}^{\circ}$ in the case of the chart plates.

6 . The meaning of the denominator may be seen most readily by taking a simple case. Let $\mathrm{C}$ be the centre of the sphere,

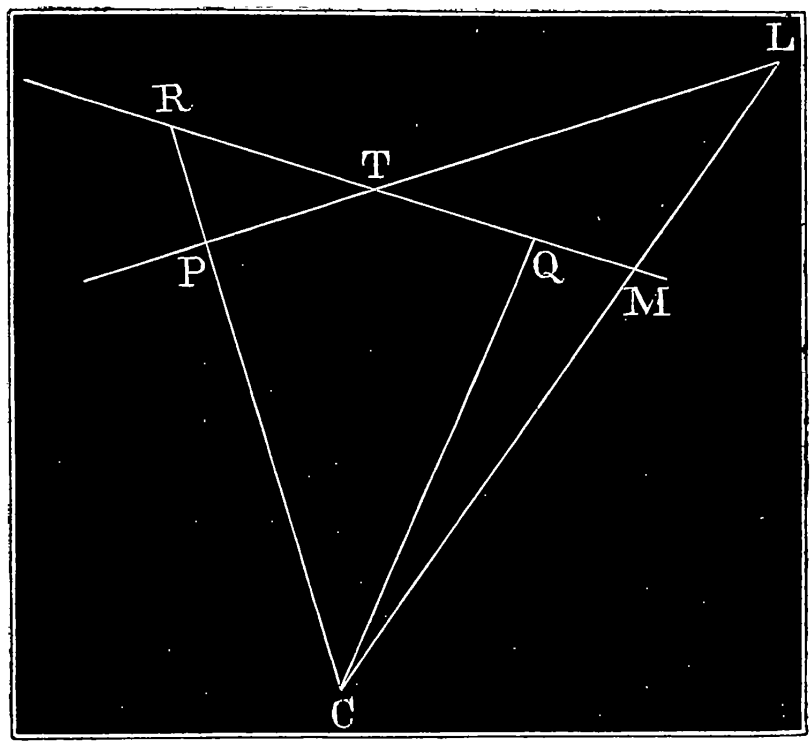

PTL, RTM two plates whose centres are P and $\mathrm{Q}$; $\mathrm{L}$ and $\mathrm{M}$ the images of a star on these plates, in a plane through PCQ; PL, QM the co-ordinates $x$ and $\xi$. Then if the radius $C P$ of the sphere be taken as unity

$$
\begin{aligned}
\xi & =\mathrm{QM}=\tan \mathrm{QCM}=\tan (\mathrm{PCL}-\mathrm{RCQ}) \\
& =\frac{\mathrm{PL}-\mathrm{RQ}}{\mathrm{I}+\mathrm{PL} \cdot \mathrm{RQ}} \\
& =\frac{x-\mathrm{RQ}}{\mathrm{I}+x \cdot \mathrm{RQ}} \\
& =\frac{x+c}{\mathrm{I}-\mathrm{k} x}
\end{aligned}
$$

where $k=c=-\mathrm{RQ}=$ the $x$ co-ordinate of centre of plate $(x, y)$ on plate $(\xi, \eta)$. And it can be shown generally that if $(x, y)$ $(\xi, \eta)$ are measured from the centres of the two plates, our general formulæ of transformation become 
Nov. I 893 .

$$
\xi=\frac{(\mathbf{I}+a) x+b y+c}{\mathbf{I}-c x-f y}, \quad \eta=\frac{d x+(\mathbf{1}+e) y+f}{\mathbf{I}-c x-f y}, *
$$

where all the quantities arc in parts of the radius. If, as is usual in measuring the Astrographic Chart plates, $x$ and $y$ (and consequently $c$ and $f$ ) be expressed in minutes (or secnnds) of arc, instead of $k=c, l=f$, we shall have

$$
k=\frac{c}{\left(343^{8}\right)^{2}} \text { or } \frac{c}{(206264)^{2}}, \& c .
$$

7. Such formulæ hold whether the co-ordinates be measured accurately in prescribed directions or not, provided they are rectilinear, but we shall often find it useful to refer to what may be called the standard co-ordinates of a plate, which are thus defined: Let $A$ and $P$ be the Right Ascension and N.P.D. for IS00.0 of the proposed centre of the plate; from this point draw two straight lines on the tangent plane t, the celestial sphere perpendicular and parallel to the meridian for $1900^{\circ} \circ$. These lines are the axes of $\xi$, and $\eta$, and $(\xi, \eta)$, the "standard co-ordinates" of any point whose R.A. and N.P.D. and $\alpha$ and $\gamma$ respectively, are expressible in terms of these quantities by the relations

where

$$
\xi=\frac{\tan (\alpha-\mathrm{A}) \sin g}{\cos (\mathrm{P}-q)}, \quad \eta=\tan (\mathrm{P}-q)
$$

$$
\tan q=\tan p \cos (\alpha-\mathrm{A}) \text {. }
$$

(See Note I.)

8. Let us now return to the disturbing causes mentioned in paragraph 2. Concerning distortion of the object-glass and displacements of the photographic film it is to be remarked, in the first place, that they have been shown to be small; and, secondly, that any correction for them falls outside this or any other general method of reduction. As regards

Refraction, rarious formulæ have been given for the differential refraction, perhaps the most elegant being those of Dr. A. A. Rambaut in Astronomische Nachrichten, No. 3125. He there shows that it is only necessary to retain terms of the first order in $\Delta \alpha \cos \delta$, and $\Delta \delta$, and finds the following expressions :-

$$
\begin{aligned}
& \Delta d \alpha=\beta_{\circ}\left(\frac{\cos \phi}{\cos \delta} \frac{\sin \nu \sin (\mu+\delta)}{\sin ^{2} n \sin ^{2}(m+\delta)} d \alpha+\frac{\cot n \cos (m+2 \delta)}{\cos ^{2} \delta \sin ^{2}(m+\delta)} d \delta\right), \\
& \Delta d \delta=\beta_{\circ}\left(\frac{\cos \phi \cos \nu}{\sin ^{2} n \cdot \sin ^{2}(m+\delta)} d \alpha+\frac{\mathbf{I}}{\sin ^{2}(m+\delta)} d \delta\right)
\end{aligned}
$$

* These formulæ are approximate only. Generally $(c, f)$ are the coordinates of centre of plate $(x, y)$ on plate $(\xi, \eta)$ and $(-k,-l)$ the co-ordinates of centre of $(\xi, \eta)$ on $(x, y)$, but in the small terms involving $(k, l)$ these are sensibly equal. (See Note IV.) 
where

$$
\begin{array}{ll}
\tan m=\cot \phi \cos (\theta-\alpha) & \cot \mu=\tan \phi \cos (\theta-\alpha) \\
\cot n=\sin m \tan (\theta-a) & \cot \nu=\cos \mu \tan (\theta-a) .
\end{array}
$$

Elegant as these formulæ are, mach simpler ones can be obtained by using rectangular co-ordinates on the plate instead of R.A. and Declination. Let $\mathrm{X}$ and $\mathrm{Y}$ be the "standard co-ordinates" (see paragraph 7) of the zenith, supposed projected on the plate. If $\lambda$ be the colatitude, $S$ the sidereal time of exposure of the plate, A and P the R.A. and N.P.D. of the centre, according to the formalæ in paragraph 7 , we have

where

$$
\mathrm{X}=\frac{\tan (\mathrm{S}-\mathrm{A}) \sin q}{\cos (\mathrm{P}-q)}, \quad \mathrm{Y}=\tan (\mathrm{P}-q),
$$

$$
\tan q=\tan \lambda \cos (S-A)
$$

These formulæ are very simple, and $q$ can readily be tabulated at any given place for different values of the hour angle $\mathrm{S}-\mathrm{A}$. Then it is shown in Note II. that the corrections for refraction become

$$
\begin{aligned}
& d \xi=\beta_{\circ}\left\{\left(\mathrm{I}+\mathrm{X}^{2}\right) \xi+\mathrm{XY} \eta\right\} \\
& d \eta=\beta_{\circ}\left\{\mathrm{XY} . \xi+\left(\mathrm{I}+\mathrm{Y}^{2}\right) \eta\right\},
\end{aligned}
$$

where $\beta_{\circ}$ is the constant of refraction, as in Dr. Rambaut's formulæ. But the point of greatest importance is the preliminary investigation of Dr. Rambaut's paper, which shows that terms of the second order in $\xi$ and $\eta$ may be neglected, and makes the above corrections linear in $\xi$ and $\eta$; for thas it follows that a straight line on the plate is still a straight line, and hence the correction for refraction is included in the general formulæ quoted above. No separate calculation of the refraction is therefore required. Similarly the correction for

Aberration is small, and all terms beyond those of the first order may be neglected. If $\left(\mathrm{X}^{\prime}, \mathrm{Y}^{\prime}\right)$ be the co-ordinates of the "Earth's Way" supposed projected on the plate, the corrections for aberration are shown in Note III. to be

$$
\begin{aligned}
& d x=\frac{\gamma_{0} x}{\sqrt{I}+\mathrm{X}^{\prime 2}+\mathrm{Y}^{\prime 2}} \\
& d y=\frac{\gamma_{0} y}{\sqrt{\mathrm{I}+\mathrm{X}^{\prime 2}+\mathrm{Y}^{\prime 2}}} .
\end{aligned}
$$

These again, being linear, are included in the general formulæ, and no separate calculation of them is required.

9. The following corrections are also well known to involve 
linear transformations only, and are similarly included in the general formulæ:-

That for orientation, whether of the plate in the telescope or in the measuring apparatus.

That for scale value, whether due to change in focal length of the telescope or in the measuring apparatus. nates.

That for non-perpendicularity of the two measured co-ordi-

Io. It follows, then, that if $(x, y)$ be the measured rectilinear co-ordinates of a star on a plate as affected by refraction, aberration, and the sources of error mentioned above; and if $(\xi, \eta)$ be its "standard co-ordinates" (see paragraph 7 ) and $\kappa$ is the number of minutes of are in the radian: then

$$
\kappa \xi=(\mathbf{I}+\alpha) x+\beta y+\gamma, \quad \kappa \eta=\delta x+(\mathbf{I}+\epsilon) y+\zeta,
$$

no denominator being required in these expressions if the theoretical and actual centres of the plate are nearly coincident. Now, if there are three known stars on the plate, we have six equations to determine the constants $a, \beta, \gamma, \delta, \epsilon, \zeta$; or, if more stars are known, we can solve by least squares or any analogous process. It is to be noted that the equations are of the linear form specially adapted to simple solution. Knowing these constants we can convert the measured co-ordinates $(x, y)$ of any star into standard co-ordinates $(\xi, \eta)$, and if desired into R.A. and N.P.D. by the formulæ of paragraph 7 : viz. find $q$ from

$$
\tan (\mathrm{P}-q)=\eta \text {, }
$$

then $a$ from

$$
\frac{\tan (\alpha-A) \sin q}{\cos (P-q)}=\xi,
$$

and finally $p$ from $\tan q=\tan p \cos (a-\mathrm{A})$. But I venture to think that this final transformation will generally be unnecessary, for the "standard co-ordinates" of a star will for many purposes be far more convenient.

II. If there be not enough known stars on the plate to give a good determination of the constants $\alpha, \beta, \gamma, \& c$., it becomes necessary to associate with it neighbouring plates in the manner suggested by M. Loewy. The process he adopts is, however, much simplified by the present method. If we have a central plate (O), and four others (I), (2), (3), (4), whose centres are approximately at the corners of the plate (o), and if suffixes denote the fact that the co-ordinates are measured on the corresponding plate, then we have 
where

$$
\begin{aligned}
& x_{\circ}=\frac{(\mathbf{I}+\alpha) x_{1}+b y_{1}+c}{\mathbf{I}-\mu c x_{1}-\mu f y_{1}} \\
& y_{\circ}=\frac{d x_{1}+(\mathbf{I}+e) y_{1}^{\prime}+f}{\mathbf{1}-\mu c x_{1}-\mu f y_{1}},
\end{aligned}
$$

$$
\mu=\frac{\mathbf{I}}{\left(343^{x}\right)^{2}}
$$

and the quantities are in minutes of arc. Since the quantities $\mu c x_{1} x_{0}, \mu f y_{1} x_{0}, \& c .$, are very small, approximate values of $c$ and $f$, such as can be found by mere inspection, wili be sufficiently accurate for substitution in these terms, and we can form the quantities

$$
\begin{aligned}
& x_{\circ}\left(\mathbf{I}-\mu c x_{1}-\mu f y_{1}\right) \\
& y_{\circ}\left(\mathbf{I}-\mu c x_{1}-\mu f y_{1}\right)
\end{aligned}
$$

for all the stars common to the two plates. We then have for each star two simple equations in $a, b, c, d, e, f$, and all these may be solved by least squares or any analogous process.

Knowing $a, b, c, d, e, f$, we can form $\left(x_{\circ}, y_{\circ}\right)$ the co-ordinates of any star $\left(x_{1} y_{1}\right)$ on the plate $(\mathrm{s})$, but outside the region common to the two plates, as though that star had been on the plate (o) and measured with the other stars on the same system. We can thus virtually transfer all the known stars on the plates (1), (2), (3), (4) on to the plate (0), and increase the number of the equations for determining $\alpha, \beta, \gamma, \& c .$, on that plate. The process is obviously capable of indefinite extension.

12. In view of this method of reduction by rectilinear coordinates, a recent decision of the Permanent Committee to publish such measures without further reduction becomes very important. It is clear that from such measures-

(I) Formulæ can quickly be deduced connecting one plate with another.

(2) The use of Right Ascensions and Declinations may be entirely deferred until we wish to deduce the final constants for any plate from known stars. Indeed, a great deal of information as to the progress of the work generally can be obtained without ever using R.A. and Declination at all, when it is once realised that measures on one plate are very simply convertible into measures on another. Rectilinear measures, instead of being very raw material, are, in fact, quite ready for use. They are readily comparable inter se, and with any similar measures of similar photographs to be made in the future. R.A. and Declination are a necessity of meridian and equatorial observation but only complicate any discussion of the places of the stars. 


\section{Note $I$.}

Let $\mathrm{O}$ be the centre of the plate, $\mathrm{K}$ and $\mathrm{L}$ its poles on the celestial sphere; $P$ the North Pole of the heavens.

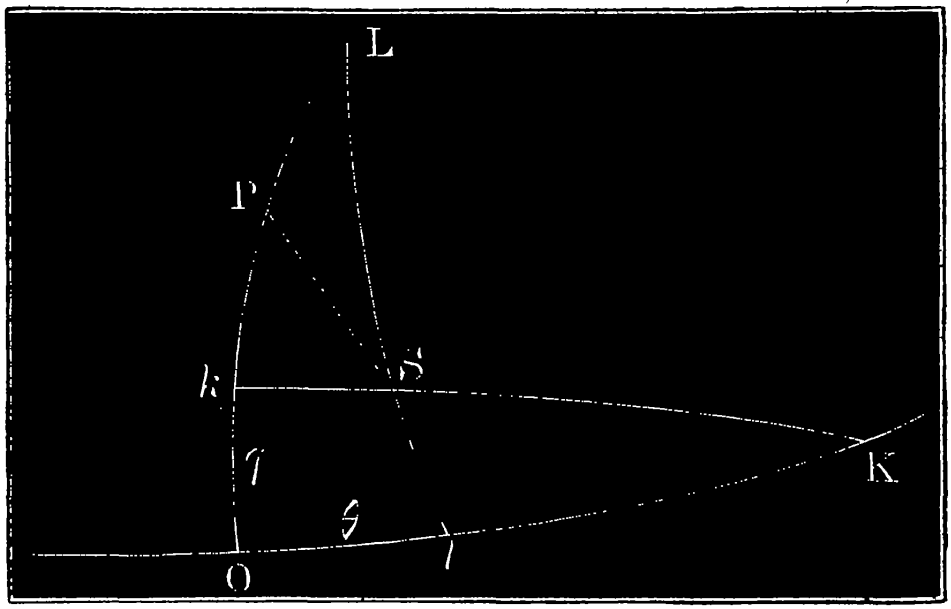

Let the R.A. of the centre $\mathrm{O}$ be A, and its N.P.D., P; and let $(\alpha, p)$ be the R.A. and N.P.D. of any star $\mathrm{S}$ on the plate.

Then

$$
\begin{aligned}
\mathrm{PS} & =p, \angle \mathrm{OPS}=a-\dot{\mathrm{A}}, \mathrm{OP}=\mathrm{P} ; \\
\xi & =\tan \mathrm{O} l=\tan \theta(\text { say) }, \eta=\tan \mathrm{O} k=\tan \phi .
\end{aligned}
$$

In the triangle LPS

$\cot \mathrm{PS} \sin \mathrm{LP}=\cos \mathrm{LP} \cos \mathrm{LPS}+\sin \mathrm{LPS} \cot \mathrm{LPS}$,

or

Thus

$$
\cot p \cos \mathrm{P}=-\sin \mathrm{P} \cos (\alpha-\mathrm{A})+\sin ^{\prime}(\alpha-\mathbf{A}) \cdot \frac{\mathbf{I}}{\xi} \cdot
$$

$$
\xi=\frac{\sin (\alpha-\mathrm{A})}{\cot p \cos \mathrm{P}+\sin \mathrm{P} \cos (\alpha-\mathrm{A})}, \quad . \quad . \quad .
$$

Let

Then

$$
\tan p \cos (\alpha-\mathrm{A})=\tan q \quad \text {. . . . . . . }
$$

$$
\begin{aligned}
\xi & =\frac{\sin (\alpha-\mathrm{A})}{\cot p(\cos \mathrm{P}+\sin \mathrm{P} \tan q)} \\
& =\frac{\sin (\alpha-\mathrm{A}) \tan p \cos q}{\cos (\mathrm{P}-q)} \\
& =\frac{\tan (\alpha-\mathrm{A}) \sin q}{\cos (\mathrm{P}-q)} . . . . . . . .
\end{aligned}
$$


Again in $\Delta k \mathrm{PS}$

or

$\cot \mathrm{PS} \sin k \mathrm{P}=\cos k \mathrm{P} \cos k \mathrm{PS}+\sin k \mathrm{PS} \cot \mathrm{P} k \mathrm{~S}$,

$$
\begin{gathered}
\cot p \sin (\mathrm{P}-\phi)=\cos (\mathrm{P}-\phi) \cos (\alpha-\mathrm{A})+\mathrm{O} ; \\
\therefore \tan (\mathrm{P}-\phi)=\cos (\alpha \quad \mathrm{A}) \tan p \\
=\tan q .
\end{gathered}
$$

Thus

$$
\eta=\tan \phi=\tan (\mathrm{P}-q) \text {. . . . . . . . }
$$

Formulæ 2, 3, 4, give $\xi$ and $\eta$ in terms of $a, p, \mathrm{~A}$, and P.

\section{Note II.}

If now $\mathrm{S}^{\prime}$ is a star on the plate, $Z$ the projection of the zenith on the plane of the plate, and $S$ be the true position of the star,

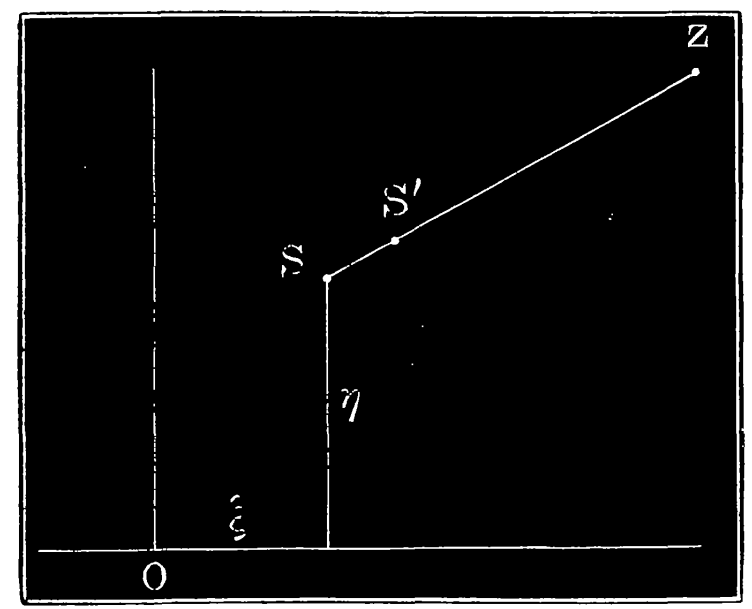

which is moved by refraction to $S^{\prime}$ on the straight line $S Z$, then since $\mathrm{SS}^{\prime}$ is sensibly equal to its arc

$$
\mathrm{SS}^{\prime}=\beta_{0} \tan \angle \mathrm{SCZ},
$$

where $\mathrm{C}$ is the centre of the sphere.

Now if

$$
\begin{aligned}
& (\mathrm{X}, \mathrm{Y}) \text { be the co-ordinates of } \mathrm{Z} \text {, } \\
& (\xi, \eta) \text { be the co-ordinates of } \mathrm{S} \text {, }
\end{aligned}
$$

by a well-known theorem of solid geometry

$$
\cos \mathrm{SCZ}=\frac{\mathbf{I}+\xi \mathrm{X}+\eta \mathrm{Y}}{\sqrt{\mathrm{I}+\xi^{2}+\eta^{2}} \sqrt{\mathbf{I}+\mathrm{X}^{2}+\mathrm{Y}^{2}}} .
$$

Dr. Rambaut has shown that it is only necessary to retain the first powers of $\xi$ and $\eta$, and hence

$$
\begin{aligned}
\cos \mathrm{SCZ} & =\frac{\mathbf{I}}{\sqrt{\mathbf{I}+\mathrm{X}^{2}+\mathrm{Y}^{2}}}\{\mathbf{I}+\xi \mathrm{X}+\eta \mathrm{Y}\} \\
& =\cos \theta_{0}\{\mathbf{I}+\xi \mathrm{X}+\eta \mathrm{Y}\},
\end{aligned}
$$

where $\theta_{0}$ is the angle OCZ. 
Nov. I 89.3.

Thas

$$
\begin{aligned}
\tan \mathrm{SC}^{\prime} L & =\tan \theta_{0}\left\{\mathrm{I}-\operatorname{cosec}^{2} \theta_{0}(\xi \mathrm{X}+\eta \mathrm{Y})\right\} \\
& =\tan \theta_{0}\left\{\mathrm{I}-\frac{\mathrm{X}^{2}+\mathrm{Y}^{2}+\mathrm{I}}{\mathrm{X}^{2}+\mathrm{Y}^{2}}(\xi \mathrm{X}+\eta \mathrm{Y})\right\} .
\end{aligned}
$$

Now the refraction resolved along $\xi$ is

$$
d \xi=\mathrm{SS}^{\prime} \frac{\mathrm{X}-\xi}{\sqrt{(\mathrm{X}-\xi)^{2}+(\overline{\mathrm{Y}}-\eta)^{2}}} ;
$$

or

$$
\begin{aligned}
d \xi & =\beta_{0} \mathrm{X} \tan \theta_{0}\left[\mathrm{I}-\frac{\mathrm{X}^{2}+\mathrm{Y}^{2}+\mathrm{I}}{\mathrm{X}^{2}+\mathrm{Y}^{2}}(\mathrm{X} \xi+\mathrm{Y} \eta)\right]\left[\mathrm{I}-\frac{\xi}{\mathrm{X}}\right]\left[\mathrm{I}+\frac{\xi \mathrm{X}+\eta \mathrm{Y}}{\mathrm{X}^{2}+\mathrm{Y}^{2}}\right] \\
& =\beta_{0} \mathrm{X}[\mathrm{I}-(\xi \mathrm{X}+\eta \mathrm{Y})]\left[\mathrm{I}-\frac{\xi}{\mathrm{X}}\right] \\
& =\beta_{0}\left[\mathrm{X}-\xi\left(\mathrm{I}+\mathrm{X}^{2}\right)-\eta \mathrm{XY}\right] .
\end{aligned}
$$

Now $\beta_{0} X$ is the refraction at the centre of the plate, and hence the differential refraction is

$$
d \xi=-\beta_{0}\left(\mathbf{I}+\mathrm{X}^{2}\right) \xi-\beta_{0} \mathrm{XY} \cdot \eta
$$

Similarly

$$
d \eta=-\beta_{0} \mathrm{XY} \xi-\beta_{0}\left(\mathbf{I}+\mathrm{Y}^{2}\right) \eta .
$$

If the photograph is taken near the meridian $X$ will be small.

The quantities $X$ and $Y$ are found by the formulæ of the preceding Note: putting $a=\mathrm{S}$, the sidereal time of exposure of the photograph, and $p=\lambda$, the colatitude.

Thus

$$
\tan q=\tan \lambda \cos (\mathrm{S}-\mathrm{A}),
$$

and can be tabulated for any given place as a simple function of the hour angle $\mathrm{S}-\mathrm{A}$. Thus :

$$
\mathrm{Y}=\tan (\mathrm{P}-q)
$$

is known at once, and

is readily found.

$$
\mathrm{X}=\frac{\tan (\mathrm{S}-\mathrm{A}) \sin q}{\cos (\mathrm{P}-q)}
$$

$\mathrm{I}+\mathrm{X}^{2}, \mathrm{XY}$, and $\mathrm{I}+\mathrm{Y}^{2}$ can be formed quickly by Crelle's tables, three figures of decimals being amply sufficient. The magnitude of the quantities can be estimated by supposing $X=0$, $\mathrm{Y}=\mathrm{I}$, i.e. a photograph taken on the meridian at $45^{\circ}$ zenith distance. Then

$$
\begin{aligned}
d \xi & =-\beta_{0} \xi \\
d \eta & =-2 \beta_{0} \eta
\end{aligned}
$$

Since $\xi$ and $\eta$ may vary from $+\mathrm{I}^{\circ}\left(\frac{\mathrm{I}}{57}\right.$ in circ. measure $)$ to $-\mathrm{I}^{\circ}\left(\right.$ or $\left.-\frac{\mathrm{I}}{57}\right)$, the corrections for refractions vary from $+\frac{\mathbf{I}}{5.7} \beta_{0}$ 
to $-\frac{\mathrm{I}}{57} \beta_{0}$ for $\xi$, and double this for $\eta$ : i.e. (since $\beta_{0}=57^{\prime \prime}$, approximately) from $+\mathrm{I}^{\prime \prime}$ to $-\mathrm{I}^{\prime \prime}$, or $+2^{\prime \prime}$ to $-2^{\prime \prime}$ respectively.

Note III.

The investigation of the correction for aberration is very similar to that for refraction. If $\mathrm{W}$ be the projection of the

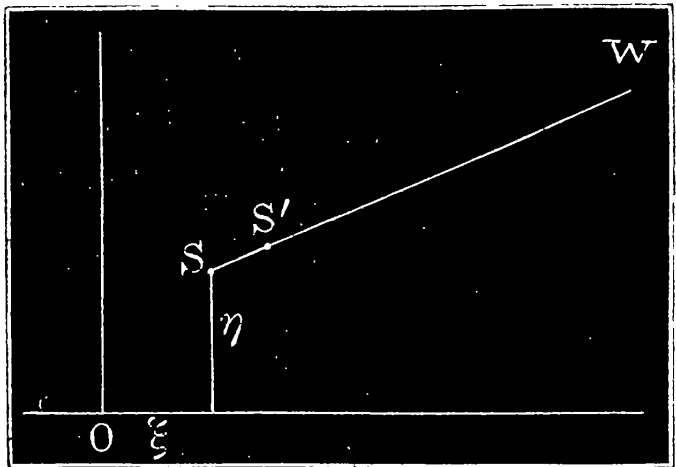

"Earth's Way" on the plate, the displacement $\mathrm{SS}^{\prime}$ is now proportional to sin SCW instead of tan SCZ.

Now

Hence

$$
\sin \mathrm{SCW}=\sin \theta_{0}\left[\mathrm{I}-\cos ^{2} \theta_{0}(\xi \mathrm{X}+\eta \mathrm{Y})\right] .
$$

$$
\begin{aligned}
d \xi & =\gamma_{0} \frac{\mathrm{X}}{\sqrt{\mathrm{X}^{2}+\mathrm{Y}^{2}}} \sin \theta_{0}\left[\mathrm{I}-\frac{\mathrm{I}}{\mathrm{X}^{2}+\mathrm{Y}^{2}}(\xi \mathrm{X}+\eta \mathrm{Y})\right]\left[\mathrm{I}-\frac{\xi}{\mathrm{X}}\right]\left[\mathrm{I}+\frac{\xi \mathrm{X}+\eta \mathrm{Y}}{\mathrm{X}^{2}+\mathrm{Y}^{\prime 2}}\right. \\
& =\gamma_{0} \mathrm{X} \sin \theta_{0} \\
\sqrt{\mathrm{X}^{2}+\mathrm{Y}^{2}} & {\left[\mathrm{I}-\frac{\xi}{\mathrm{X}}\right] } \\
& =\gamma_{0} \frac{\mathrm{X}}{\sqrt{\mathrm{I}+\mathrm{X}^{2}+\mathrm{Y}^{2}}}-\sqrt{\sqrt{\mathrm{I}+\mathrm{X}^{2}+\mathrm{Y}^{2}}}
\end{aligned}
$$

The first term is the aberration at the centre of the plate. Thus the differential aberration is

$$
\begin{aligned}
& d \xi=-\frac{\gamma_{0} \xi}{\sqrt{\mathrm{I}+\mathrm{X}^{2}+\mathrm{Y}^{2}}} \cdot \cdot \cdot \cdot \cdot \cdot \cdot \cdot \cdot \cdot(7) \\
& d \eta=-\frac{\gamma_{0} \eta}{\sqrt{\mathrm{I}+\mathrm{X}^{2}+\mathrm{Y}^{2}}}
\end{aligned}
$$

where $\gamma_{0}$ is the coefficient of aberration $=20^{\prime \prime} \cdot 4$ and $(\mathrm{X}, \mathrm{Y})$ is the projection of the "Earth's Way" on the plane of the plate.

\section{Note IV.}

On the Relations between, the Co-ordinates of the same object on two Different Plates.

Let us first consider the relations between two sets of "Standard" co-ordinates, i.e. co-ordirates accurately perpendicular and 
Nor. 1893.

parallel to the meridian at the centre of the plate, expressed in circular measure. Let us call the two plates (I) and (2), and let the R.A.'s of their centres be $A_{1}$ and $A_{2}$, the N.P.D.'s being $\mathrm{P}_{1}$ and $\mathrm{P}_{2}$. Let $\left(x_{1}, y_{1}\right)$ be the co-ordinates of a point whose R.A. and N.P.D. are $(a, p)$ on plate (r), and $\left(x_{2}, y_{2}\right)$ its co-ordinates on plate (2).

Then from Note I.

$$
x_{1}=\frac{\tan \left(\alpha-\mathrm{A}_{1}\right) \sin }{\cos \left(\mathrm{P}_{1}-q_{1}\right)}, \quad q_{1}=\tan \left(\mathrm{P}_{1}-q_{1}\right),
$$

where

$$
\tan q_{1}=\tan p \cos \left(\alpha-A_{1}\right),
$$

or more conveniently for our present purpose since

$$
\begin{aligned}
\tan \left(\alpha-\mathrm{A}_{1}\right) & =\sin \left(\alpha-\mathrm{A}_{1}\right) \cdot \frac{\sin q_{1}}{\cos \left(\alpha-\mathrm{A}_{1}\right)} \\
& =\sin \left(\alpha-\mathrm{A}_{1}\right) \tan p \cos q_{1} ;
\end{aligned}
$$

bence dividing both numerator and denominator of the expression for $x_{1}$ by $\cos q_{1} \cos \mathrm{P}_{1}$

$$
x_{1}=\frac{\tan p \sin \left(\alpha-\mathrm{A}_{1}\right) \sec \mathrm{P}_{1}}{\mathrm{I}+\tan p \tan \mathrm{P}_{1} \cos \left(\alpha-\mathrm{A}_{1}\right)}
$$

and

Thus

$$
y_{1}=\frac{\tan \mathrm{P}_{1}-\tan p \cos \left(\alpha-\mathrm{A}_{1}\right)}{\mathrm{i}+\tan p \tan \mathrm{P}_{1} \cos \left(\alpha-\mathrm{A}_{1}\right)}
$$

and therefore

$$
\mathbf{I}+y_{1} \tan \mathrm{P}_{1}=\frac{\sec ^{2} \mathrm{P}_{1}}{\mathrm{I}+\tan p \tan \mathrm{P}_{1} \cos \left(\alpha-\mathbf{A}_{\mathbf{1}}\right)}
$$

$$
\begin{aligned}
& \tan p \sin \left(\alpha-\mathrm{A}_{1}\right)=\frac{x_{1} \sec \mathrm{P}_{1}}{\mathrm{I}+y_{1} \tan \overline{\mathrm{P}}_{1} ;} \\
& \tan p \cos \left(\alpha-\mathrm{A}_{1}\right)=\frac{\tan \mathrm{P}_{1}-y_{1}}{\mathrm{I}+y_{1} \tan \mathrm{P}_{1}} .
\end{aligned}
$$

Thas we have, since

writing

$$
x_{2}=\frac{\tan p \sin \left(\alpha-\mathrm{A}_{2}\right) \sec \mathrm{P}_{2}}{\mathrm{I}+\tan p \tan \mathrm{P}_{2} \cos \left(\alpha-\mathrm{A}_{2}\right)} \& \mathrm{c} .
$$

$$
\left(\alpha-A_{2}\right)=\left(\overline{\alpha-A_{1}}+\overline{A_{1}-A_{2}}\right)
$$

expanding the sine and cosine of the expression, and substituting the values of $\tan p \sin \left(a-A_{1}\right)$ and $\tan p \cos \left(a-A_{1}\right)$, from above, $x_{2}=\frac{x_{1} \sec \mathrm{P}_{1} \sec \mathrm{P}_{2} \cos \overline{\mathrm{A}_{1}-\mathrm{A}_{2}}+\left(\tan \mathrm{P}_{1}-y_{1}\right) \sec \mathrm{P}_{2} \sin \overline{\mathrm{A}_{1}-\mathrm{A}_{2}}}{\mathrm{I}+y_{1} \tan \mathrm{P}_{1}+\tan \mathrm{P}_{2}\left(\tan \mathrm{P}_{1}-y_{1}\right) \cos \overline{\mathrm{A}_{1}-\mathrm{A}_{2}-x_{1} \tan \mathrm{P}_{2} \sec \mathrm{P}_{1} \sin \overline{\mathrm{A}_{1}-\mathrm{A}_{2}}}}$ and similarly

$$
y_{2}=\frac{\tan \mathrm{P}_{2}\left(\mathbf{I}+y_{1} \tan \mathrm{P}_{1}\right)-\left(\tan \mathrm{P}_{1}-y_{1}\right) \cos \overline{\mathrm{A}_{1}-\mathrm{A}_{2}}+x_{1} \sec \mathrm{P}_{1} \sin \overline{\mathrm{A}_{1}-\overline{\mathbf{A}}_{2}}}{\text { silme denominator }}
$$


Now let $\left(\mathrm{X}_{12}, \mathrm{Y}_{12}\right)$ be the co-ordinates of the centre of plate (I) on plate (2). Then putting $x_{1}=y_{1}=0$ in the above expressions we have

$$
\begin{aligned}
& X_{12}=\frac{\tan P_{1} \sec P_{2} \sin \overline{A_{1}-A_{2}}-}{I+\tan P_{1} \tan P_{2} \cos \overline{A_{1}-A_{2}}} ; \\
& Y_{12}=\frac{\tan P_{2}-\tan P_{1} \cos \overline{A_{1}-A_{2}}}{I+\tan P_{1} \tan P_{2} \cos \overline{A_{1}-A_{2}}} .
\end{aligned}
$$

Returning now to the expressions for $x_{2}$ and $y_{2}$, consider first the common denominator. Dividing throughout by the constant term $\mathrm{I}+\tan \mathrm{P}_{1} \tan \mathrm{P}_{2} \cos \overline{\mathrm{A}_{1}-\mathrm{A}_{2}}$, which we may call $\mathrm{D}$, the constant term becomes unity; the coefficient of $x_{1}$ becomes

$$
\left(-\tan P_{2} \sec P_{1} \sin \overline{A_{1}-A_{2}}\right) / D
$$

(which by comparison with the expression just obtained for $\mathrm{X}_{12}$ we see to be $X_{21}$ ), and the coefficient of $y_{1}$ becomes

$$
\left(\tan P_{1}-\tan P_{2} \cos \overline{A_{1}-A_{2}}\right) / D \text {, }
$$

which is thus equal to $\mathrm{Y}_{21}$.

Hence the denominator becomes

$$
\mathrm{I}+x_{1} \mathrm{X}_{21}+y_{1} \mathrm{Y}_{21} \text {. }
$$

In the numerator of the expression for $x_{2}$ the constant term is, of course, $X_{12}$.

The coefficients of $x_{1}$ and $y_{1}$ in the numerators of the expressions for $x_{2}$ and $y_{2}$ can be simplified by the use of the quantities $\mathrm{X}_{12} \mathrm{Y}_{12}$ or $\mathrm{X}_{21} \mathrm{Y}_{21}$. The expressions for these quantities in terms of $P_{1}, P_{2}$, and $\overline{A_{1}-A_{2}}$ give four equations from which $\mathrm{P}_{1}, \mathrm{P}_{2}$, and $\overline{\mathrm{A}_{1}-\mathrm{A}_{2}}$ can be eliminated, leaving one relation between the four quantities $X_{12}, Y_{12}, X_{21}, Y_{21}$. Without performing the elimination directly it is easily seen that this relation is

$$
\mathrm{X}_{12}^{2}+\mathrm{Y}_{12}^{2}=\mathrm{X}_{21}^{2}+\mathrm{Y}_{21}^{2} \text {. . . . . . . . (9) }
$$

each side of the equation being the square of the projection of the are joining the two centres on the tangent planes at the extremities of that arc.

Further

$$
\mathrm{X}_{12} \sin \mathrm{P}_{2}+\mathrm{X}_{21} \sin \mathrm{P}_{1}=0 \text {. . . . . . . }
$$

and

$$
\begin{aligned}
& \quad D\left(Y_{12} \sin P_{2} \cos P_{2}-Y_{21} \sin P_{1} \cos P_{1}\right) \\
& =\left(\sin ^{2} P_{2}-\sin ^{2} P_{1}\right)+\sin P_{1} \sin P_{2} \cos \overline{A_{1}-A_{2}}\left(\frac{\cos P_{1}}{\cos P_{2}}-\frac{\cos P_{2}}{\cos P_{1}}\right) \\
& =\left(\operatorname{sil}^{2} P_{2}-\sin ^{2} P_{1}\right) D .
\end{aligned}
$$


Nov. 1893.

Thus

$$
\mathrm{Y}_{12} \sin 2 \mathrm{P}_{2}-\mathrm{Y}_{21} \sin 2 \mathrm{P}_{1}=\cos 2 \mathrm{P}_{1}-\cos 2 \mathrm{P}_{2} \text {. }
$$

These three relations independent of ${\overline{A_{2}-A_{1}}}_{1}$ are obviously all that can be obtained from the above four equations.

Again, we have

Thus

$$
\begin{aligned}
& \mathrm{D}=\mathrm{I}+\tan \mathrm{P}_{1} \tan \mathrm{P}_{2} \cos {\overline{A_{1}-A_{2}}}_{2}=\frac{\sec ^{2} \mathrm{P}_{2}}{\mathrm{I}+\mathrm{Y}_{12} \tan \mathrm{P}_{2}} \\
& \tan P_{1} \tan P_{2} \cos {\overline{A_{1}-A_{2}}}_{2}=\frac{\tan P_{2}\left(\tan P_{2}-Y_{12}\right)}{I+Y_{12} \tan P_{2}} \\
& \tan P_{1} \tan P_{2} \sin \overline{A_{1}-A_{2}}=\frac{X_{12} \tan P_{2} \sec P_{1}}{I+Y_{12} \tan P_{2}}
\end{aligned}
$$

Hence in the numerator of the expression for $x_{2}$ the coefficient of $x_{1}$ is

$$
\begin{aligned}
& \left(\sec P_{1} \sec P_{2} \cos \overline{A_{1}-A_{2}}\right) / D \\
& =\frac{\tan P_{2}\left(\tan P_{2}-Y_{12}\right)}{\sin P_{1} \sin P_{2} \sec ^{2} P_{2}} \\
& =\left(\tan P_{2}-Y_{12}\right) \cos P_{2} \operatorname{cosec} P_{1}
\end{aligned}
$$

We can give this a more symmetrical form by the use of the relation

viz.

$$
\cos 2 \mathrm{P}_{1}-\cos 2 \mathrm{P}_{2}=\mathrm{Y}_{12} \sin 2 \mathrm{P}_{2}-\mathrm{Y}_{21} \sin 2 \mathrm{P}_{1}
$$

$$
\begin{gathered}
\frac{\sin \mathrm{P}_{2}\left(\mathrm{Y}_{12} \sin 2 \mathrm{P}_{2}-\mathrm{Y}_{21} \sin 2 \mathrm{P}_{1}\right)-\mathrm{Y}_{12} \cos \mathrm{P}_{2}\left(\cos 2 \mathrm{P}_{1}-\cos 2 \mathrm{P}_{2}\right)}{\sin \mathrm{P}_{1}\left(\mathrm{Y}_{12} \sin 2 \mathrm{P}-\mathrm{Y}_{21} \sin 2 \mathrm{P}_{1}\right)} \\
=\frac{\left.\mathrm{Y}_{12} \cos \left(2 \mathrm{P}_{2}-\mathrm{P}_{2}\right)-\mathrm{Y}_{12} \cos \mathrm{P}_{2} \cos 2 \mathrm{P}_{1}\right)-\mathrm{Y}_{21} \sin \mathrm{P}_{2} \sin 2 \mathrm{P}_{1}}{\sin \mathrm{P}_{1}\left(\mathrm{Y}_{12} \sin 2 \mathrm{P}_{2}-\mathrm{Y}_{21} \sin 2 \mathrm{P}_{1}\right)} \\
=\frac{\mathrm{Y}_{12} \cos \mathrm{P}_{2} \sin \mathrm{P}_{1}-\mathrm{Y}_{21} \sin \mathrm{P}_{2} \cos \mathrm{P}_{1}}{\mathrm{Y}_{12} \cos \mathrm{P}_{2} \sin \mathrm{P}_{2}-\mathrm{Y}_{21} \sin \mathrm{P}_{1} \cos \mathrm{P}_{1}} . . . . . . .
\end{gathered}
$$

The coefficient of $y_{1}$ in the numerator of $x_{2}$ is

$$
\begin{aligned}
& \left(-\sec \mathrm{P}_{2} \sin \overline{\mathrm{A}_{1}-\mathrm{A}_{2}}\right) \mathrm{D} \\
= & -\mathrm{X}_{12} \operatorname{cosec} \mathrm{P}_{1} \sec \mathrm{P}_{2} \cos ^{2} \mathrm{P}_{2} \\
= & -\mathrm{X}_{12} \frac{\cos \mathrm{P}_{2}}{\sin \mathrm{P}_{1}} \text { or } \mathrm{X}_{21} \cot \mathrm{P}_{2} \text {. ........ }
\end{aligned}
$$

The coefficient of $x_{1}$ in the numerator of $y_{2}$ is

$$
\begin{gathered}
\left(\sec P_{1} \sin \overline{A_{1}-A_{2}}\right) D \\
=X_{12} \cot P_{1} .
\end{gathered}
$$

The coefficient of $y_{1}$ in the numerator of $y_{2}$ is

$$
\left\{\tan P_{1} \tan P_{2}+\cos A_{1}-A_{2}\right\} \div D
$$

$$
\begin{aligned}
& =\tan P_{1} \tan P_{2} \cos ^{2} P_{2}\left(I+Y_{12} \tan P_{2}\right)+\cot P_{1} \cos ^{2} P_{2}\left(\tan P_{2}-Y_{12}\right) \\
& =\sin \mathrm{P}_{2} \cos \mathrm{P}_{2}\left(\tan \mathrm{P}_{1}+\cot \mathrm{P}_{1}\right)+\mathrm{Y}_{12}\left(\sin ^{2} \mathrm{P}_{2} \tan \mathrm{P}_{1}-\cos ^{2} \mathrm{P}_{2} \cot \mathrm{P}_{1}\right) \\
& =\frac{\sin 2 \mathrm{P}_{2}}{\sin 2 \mathrm{P}_{1}}+\frac{\mathrm{I}}{2} \mathrm{Y}_{12}\left\{\left(\tan \mathrm{P}_{1}-\cot \mathrm{P}_{1}\right)-\cos 2 \mathrm{P}_{2}\left(\tan \mathrm{P}_{1}+\cot \mathrm{P}_{1}\right)\right\} \\
& =\frac{\sin 2 \mathrm{P}_{2}}{\sin 2 \mathrm{P}_{1}}-\mathrm{Y}_{12}\left\{\frac{\cos 2 \mathrm{P}_{1}+\cos 2 \mathrm{P}_{2}}{\sin 2 \mathrm{P}_{\mathrm{i}}}\right\}
\end{aligned}
$$


and by means of the relation

$$
\cos 2 \mathrm{P}_{1}-\cos 2 \mathrm{P}_{2}=\mathrm{Y}_{12} \sin 2 \mathrm{P}_{2}-\mathrm{Y}_{21} \sin 2 \mathrm{P}_{1}
$$

this becomes

$$
\frac{\mathrm{Y}_{12} \sin 2 \mathrm{P}_{1}-\mathrm{Y}_{21} \sin 2 \mathrm{P}_{2}}{\mathrm{Y}_{12} \sin 2 \mathrm{P}_{2}-\mathrm{Y}_{21} \sin 2 \mathrm{P}_{1}} \text {. . . . . . . . }
$$

Collecting the results, we have

Numerator of $x_{2}$

$$
\begin{aligned}
& \left.\begin{array}{rl}
= & x_{1} \cdot \frac{\mathrm{Y}_{12} \cos \mathrm{P}_{2} \sin \mathrm{P}_{1}-\mathrm{Y}_{21} \sin \mathrm{P}_{2} \cos \mathrm{P}_{1}}{\mathrm{Y}_{12} \cos \mathrm{P}_{2} \sin \mathrm{P}_{2}-\mathrm{Y}_{21} \sin \mathrm{P}_{1} \cos \mathrm{P}_{1}}+y_{1} \cot \mathrm{P}_{2}+\mathrm{X}_{12} \cdot \\
& \text { Numerator of } y_{2} \\
= & x_{1} \mathrm{X}_{12} \cot \mathrm{P}_{1}+y_{1} \cdot \frac{\mathrm{Y}_{12} \cos \mathrm{P}_{1} \sin \mathrm{P}_{1}-\mathrm{Y}_{21} \sin \mathrm{P}_{2} \cos \mathrm{P}_{2}}{\mathrm{Y}_{12} \cos \mathrm{P}_{2} \sin \mathrm{P}_{2}-\mathrm{Y}_{21} \sin \mathrm{P}_{1} \cos \mathrm{P}_{1}}+\mathrm{Y}_{12}
\end{array}\right\} . \\
& \text { Common denominator } \\
& =\mathrm{I}+x_{1} \mathrm{X}_{21}+y_{1} \mathrm{Y}_{21} \text {. }
\end{aligned}
$$

The expressions for the coefficients of $x_{1}$ in $x_{2}$, and $y_{1}$ in $y_{8}$, are, however, inconvenient; for when

$$
\mathrm{P}_{1}=\mathrm{P}_{2}, \quad \mathrm{Y}_{12}=\mathrm{Y}_{21} \text {, }
$$

and they assume the indeterminate form $\stackrel{\circ}{\circ}$.

Hence when $P_{1}$ is nearly equal to $P_{2}$ they are not adapted to accurate calculation. The unsymmetrical expressions

and

$$
\frac{\sin \mathrm{P}_{2}}{\sin \mathrm{P}_{1}}-\mathrm{Y}_{12} \frac{\cos \mathrm{P}_{2}}{\sin \mathrm{P}_{1}}
$$

$$
\frac{\sin 2 \mathrm{P}_{2}}{\sin 2 \mathrm{P}_{1}}-\mathrm{Y}_{12}\left\{\frac{\cos 2 \mathrm{P}_{1}+\cos 2 \mathrm{P}_{2}}{\sin 2 \mathrm{P}_{1}}\right\}
$$

are better adapted for this purpose.

The above expressions are all rigorous. When $P_{1}$ and $P_{2}$ are not too small the coefficients of $x_{1}$ and $y_{1}$ in the numerators of $x_{2}$ and $y_{2}$ respectively are unity, to the first order of small quantities; and we bave

$$
\begin{aligned}
& \Delta x_{2}=x_{1}+y_{1} \mathrm{X}_{21} \cot \mathrm{P}_{2}+\mathrm{X}_{12} \\
& \Delta y_{2}=x_{1} \mathrm{X}_{12} \cot \mathrm{P}_{1}+y_{1}+\mathrm{Y}_{12}
\end{aligned}
$$

where

$$
\Delta=\mathbf{I}+x_{1} \mathrm{X}_{21}+y_{1} \mathrm{Y}_{21}
$$

and all the quantities are in circular measure.

Secondly, consider the relations between actually measured co-ordinates on actual plates. These will only approximately 
fulfil the theoretical conditions, owing to errors introduced by refraction, aberration, centreing, orientation, and scale values. All these should be very small, however; and if the co-ordinates $\left(x_{1} y_{1}\right)$ are corrected by the above formulæ they should agree very nearly with $\left(x_{2} y_{2}\right)$-within a few seconds of arc, say $10^{\prime \prime}$. Let their corrected values be called $\xi_{2}$ and $\eta_{2}$.

Then

$$
\begin{aligned}
& x_{2}=(\mathbf{I}+a) \xi_{2}+b \eta_{2}+c \\
& y_{2}=d \xi_{2}+(\mathbf{I}+e) \eta_{2}+f,
\end{aligned}
$$

where $a b c d$ ef are very small quantities of the order $\frac{10^{\prime \prime}}{60^{\prime}}$ or $\frac{x}{360}$, whose squares and products only introduce errors of the order

$$
60^{\prime} \times\left(\frac{\mathrm{I}}{360}\right)^{2}=0^{\prime \prime} \cdot 03
$$

and may therefore be neglected. Hence if we have a chain of plates each overlapping the next, and if we first correct the co-ordinates of one by the theoretical expressions which should make them agree with those of the next, the differences between the co-ordinates of a star $x_{1}-x_{2}, y_{1}-y_{2}$ will be linear functions of $x$ and $y$ with very small coefficients, say

$$
\begin{aligned}
& x_{1}-x_{2}=a_{12} x_{1}+b_{12} y_{1}+c_{12} \\
& y_{1}-y_{2}=d_{12} x_{1}+e_{12} y_{1}+f_{12},
\end{aligned}
$$

and it is easily seen that

$$
a_{1 n}=a_{12}+a_{23}+\ldots+a_{\overline{n-1} \cdot n},
$$

and similarly for the other coefficients. Any system of plates which returns to the same plate thus gives us 6 equations between the experimentally determined coefficients of relation between each two.

Greenwich Mean 'Times of Superior and Inferior Geocentric Conjunctions of Jupiter's Satellite Cullisto from 1894 January to 1895 February.

(Communicated by the Superintendent of the Nautical Almanac.)

The following times of geocentric conjunction of Callisto are in continuation of those published in Monthly Notices, vol. lii. p. 597 , and are communicated to the Society with the view of drawing the attention of observers to the desirability of securing determinations of the positions of this satellite during the period 